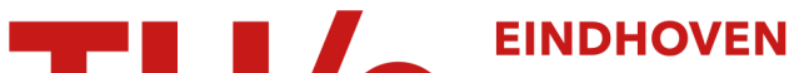 UNIVERSITY OF TECHNOLOGY
}

\section{Scanning tunneling spectroscopy on organic semiconductors : experiment and model}

\section{Citation for published version (APA):}

Kemerink, M., Alvarado, S. F., Müller, P., Koenraad, P. M., Salemink, H. W. M., Wolter, J. H., \& Janssen, R. A. J. (2004). Scanning tunneling spectroscopy on organic semiconductors : experiment and model. Physical Review B, 70(4), 045202-1/13. [045202]. https://doi.org/10.1103/PhysRevB.70.045202

DOI:

10.1103/PhysRevB.70.045202

Document status and date:

Published: 01/01/2004

\section{Document Version:}

Publisher's PDF, also known as Version of Record (includes final page, issue and volume numbers)

\section{Please check the document version of this publication:}

- A submitted manuscript is the version of the article upon submission and before peer-review. There can be important differences between the submitted version and the official published version of record. People interested in the research are advised to contact the author for the final version of the publication, or visit the $\mathrm{DOI}$ to the publisher's website.

- The final author version and the galley proof are versions of the publication after peer review.

- The final published version features the final layout of the paper including the volume, issue and page numbers.

Link to publication

\section{General rights}

Copyright and moral rights for the publications made accessible in the public portal are retained by the authors and/or other copyright owners and it is a condition of accessing publications that users recognise and abide by the legal requirements associated with these rights.

- Users may download and print one copy of any publication from the public portal for the purpose of private study or research.

- You may not further distribute the material or use it for any profit-making activity or commercial gain

- You may freely distribute the URL identifying the publication in the public portal.

If the publication is distributed under the terms of Article 25fa of the Dutch Copyright Act, indicated by the "Taverne" license above, please follow below link for the End User Agreement:

www.tue.nl/taverne

Take down policy

If you believe that this document breaches copyright please contact us at:

openaccess@tue.nl

providing details and we will investigate your claim. 


\title{
Scanning tunneling spectroscopy on organic semiconductors: Experiment and model
}

\author{
M. Kemerink \\ Molecular Materials and Nano-systems, Departments of Applied Physics and Chemical Engineering, \\ Eindhoven University of Technology, P.O. Box 513, 5600 MB, Eindhoven, The Netherlands \\ S. F. Alvarado and P. Müller \\ IBM Research, Zurich Research Laboratory, Säumerstrasse 4, CH-8803, Rüschlikon, Switzerland \\ P. M. Koenraad, H. W. M. Salemink, and J. H. Wolter \\ COBRA Inter-University Research Institute, Eindhoven University of Technology, P.O. Box 513, 5600 MB, \\ Eindhoven, The Netherlands \\ R. A. J. Janssen \\ Molecular Materials and Nano-systems, Departments of Applied Physics and Chemical Engineering, \\ Eindhoven University of Technology, P.O. Box 513, 5600 MB, Eindhoven, The Netherlands \\ (Received 1 September 2003; revised manuscript received 17 February 2004; published 9 July 2004)
}

\begin{abstract}
Scanning-tunneling spectroscopy experiments performed on conjugated polymer films are compared with three-dimensional numerical model calculations for charge injection and transport. It is found that if a sufficiently sharp tip is used, the field enhancement near the tip apex leads to a significant increase in the injected current, which can amount to more than an order of magnitude and can even change the polarity of the predominant charge carrier. We show that when charge injection from the tip into the organic material predominates, it is possible to probe the electronic properties of the interface between the organic material and a metallic electrode directly by means of tip height versus bias voltage measurements. Thus, one can determine the alignment of the molecular orbital energy levels at the buried interface, as well as the single-particle band gap of the organic material. By comparing the single-particle energy gap and the optical absorption threshold, it is possible to obtain an estimate of the exciton binding energy. In addition, our calculations show that by using a one-dimensional model, reasonable parameters can only be extracted from $z-V$ and $I-V$ curves if the tip apex radius is much larger than the tip height. In all other cases, the full three-dimensional problem needs to be considered.
\end{abstract}

DOI: 10.1103/PhysRevB.70.045202

PACS number(s): 71.38.-k, 73.61.Ph, 68.37.Ef

\section{INTRODUCTION}

Since the pioneering work of Benjamin Franklin on lightning rods in 1752, it has been known that electric fields are enhanced at the apex of sharp metallic objects. Nowadays, scanning-tunneling microscope (STM) tips with apex radii below $10 \mathrm{~nm}$ are routinely made, ${ }^{1,2}$ and it is logical to expect that geometry-induced field enhancement can play a large role in STM experiments. However, the subject has received relatively little attention. ${ }^{3-5}$ The reason for this seems to be that, for tunneling to most common samples, i.e., metals and inorganic semiconductors, geometry effects seem to be small when the tip apex radius is larger than one or a few $^{3}$ nanometers. Regarding charge injection, the sharpness of the tip apex becomes extremely important when the tip makes physical contact with a semiconducting material. In this case, the tunnel barrier collapses and a potential barrier at the metal/semiconductor interface can form. As a result, the applied potential difference drops completely within the semiconducting material, and charge-carrier injection into and transport of charge carriers within the bulk of the semiconductor are strongly influenced by the shape of the tip. Contact-mode tip displacement versus bias voltage $(z-V)$ spectroscopy at a constant current has been demonstrated as a potential technique to probe the electronic properties of buried organic/metal interfaces, ${ }^{6,7}$ as well as the charge transport within organic materials. ${ }^{8,9}$ Here, we present the results of modeling calculations for charge injection from a sharp metallic tip into semiconducting organic materials.

Using a three-dimensional numerical model, it will be shown that for carrier injection and transport, geometry effects are crucial in both current-voltage $(I-V)$ and tip heightvoltage $(z-V)$ spectroscopy. Moreover, we will show that some details of the electronic structure, i.e., the singleparticle, or polaronic, band gap, and the alignment of the molecular orbital energy levels at the organic-metal interface at both tip and substrate, can be extracted from $z-V$ curves, provided a sufficiently sharp tip is used for the measurements. We note that knowledge of the single-particle gap is particularly interesting because an estimate of the exciton binding energy $E_{\mathrm{b}}$ can then be obtained by using the relation $E_{\mathrm{b}}=E_{\mathrm{g}, \mathrm{sp}}-E_{\mathrm{a}}$, where $E_{\mathrm{a}}$ is the optical band gap. Our results extend and unify earlier interpretations of experimental data that are valid for either a very $\operatorname{sharp}^{6}$ or a relatively blunt tip. ${ }^{8}$

The relevance of these results is emphasized by the fact that many organic semiconductors exhibit a strong tendency to form highly inhomogeneous layers. ${ }^{10-12}$ As the length scale of these inhomogeneities is typically between a few nanometers and $1 \mu \mathrm{m},{ }^{10-12}$ scanning probes are essential 

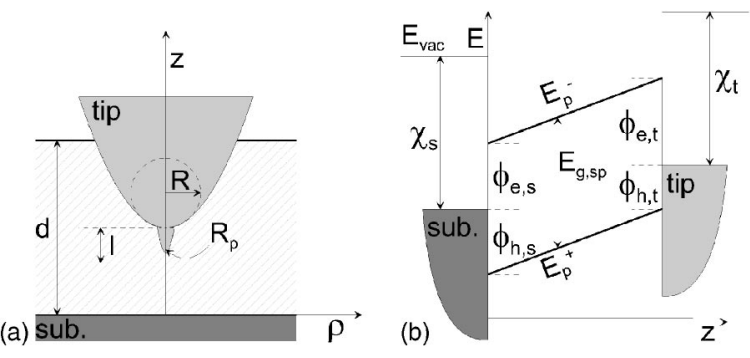

FIG. 1. Illustration of (a) the geometry and (b) energy diagram showing the parameters describing the band alignment at the organic/metal interface used for the model calculations. The shaded parabolas symbolize the density of states of the metallic electrodes.

tools in the study of organic semiconductor thin films.

The remainder of this paper is organized as follows: the numerical model will be outlined in Sec. II, and experimental details will be summarized briefly in Sec. III. Then, experimental data will be discussed and compared with model calculations in Sec. IV. Various aspects of the modeling results will be discussed in more detail in Sec. V, and Sec. VI summarizes our findings.

\section{MODEL}

The numerical model outlined below describes the simultaneous electron and hole transport through a layer of organic semiconductor material, embedded between two metallic electrodes, see Fig. 1(a). The tip shape is approximated by a paraboloid with radius of curvature $R$ at its apex. In addition, a parabolic protrusion, of length $l$ and radius of curvature $R_{\mathrm{p}}$, may be present at the tip apex. The formation of a "nanotip" at the apex of a larger "microscopic" tip by sputtering under ultrahigh-vacuum (UHV) conditions has been reported in Refs. 1 and 13. To reduce the numerical effort, rotational symmetry about the $z$ axis and a planar substrate are assumed. The substrate is defined at $z=0$, making $z$ the distance between tip apex and substrate. Both tip and substrate are treated as free-electron metals with work functions $\chi_{\mathrm{t}}$ and $\chi_{\mathrm{s}}$, respectively [Fig. 1(b)]. The organic material is treated as a jelly, in the sense that all internal inhomogeneities due to the presence of individual molecules or polymer chains are ignored. As the dimensions of the contact areas are typically much larger than the length scale of these inhomogeneities-both tip and substrate contact many molecules-this assumption is reasonable. Only in wellcontrolled situations, which require at least the presence of a vacuum gap, can atomistic models for carrier injection become meaningful. ${ }^{14}$ In addition, tip movement during $z-V$ spectroscopy is assumed to be slow enough to allow the organic layer to equilibrate.

The energy difference between the positively and negatively charged polaronic states, $E_{p}^{+}$and $E_{P}^{-}$, is equal to the single-particle gap $E_{\mathrm{g}, \mathrm{sp}}$. The barriers for electron and hole injection at each contact are $\phi_{\mathrm{e}, \mathrm{t}}$ and $\phi_{\mathrm{h}, \mathrm{t}}$ for the tip and $\phi_{\mathrm{e}, \mathrm{s}}$ and $\phi_{\mathrm{h}, \mathrm{s}}$ for the substrate, respectively, see Fig. 1(b). Note that $\phi_{\mathrm{h}, \mathrm{s}}\left(\phi_{\mathrm{e}, \mathrm{s}}\right)$ is the energy difference between $E_{P}^{+}\left(E_{P}^{-}\right)$and the substrate Fermi level. Charge transport is assumed to occur among localized sites, with a Gaussian distribution of site energies $E$ (Refs. 15 and 16) according to

$$
g(E)=g_{0}\left(2 \pi \Gamma^{2}\right)^{-1 / 2} \exp \left(-\frac{E^{2}}{2 \Gamma^{2}}\right),
$$

where $g_{0}$ is the total density of states and $\Gamma$ is the level broadening.

The transport of carriers through the organic material is described as a two-step process: First, injection from the metallic contact occurs into a localized site, i.e., negative $\left(E_{P}^{-}\right)$ and positive $\left(E_{P}^{+}\right)$polaron levels, of the organic material, ${ }^{17}$ and subsequent (bulk) transport of the carriers towards the collecting electrode (note: energies are referred to the Fermi energy of the substrate). In this way, no a priori assumptions need to be made about the predominance of one of the two processes. The injection of carriers into the organic material is described by the hopping-injection model of Arkhipov et al. ${ }^{18}$ which was recently shown to yield accurate results for similar materials as the ones used here. ${ }^{19}$ The injectioncurrent density $j_{\text {inj }}$ is written as

$$
\begin{aligned}
j_{\mathrm{inj}}= & e \nu_{0} \int_{a_{\mathrm{NN}}}^{\infty} d x_{0} \exp \left(-2 \kappa x_{0}\right) w_{\mathrm{esc}}\left(x_{0}\right) \\
& \times \int_{-\infty}^{\infty} d E^{\prime} \operatorname{Bol}\left(E^{\prime}\right) g\left[e U\left(x_{0}\right)-E^{\prime}\right],
\end{aligned}
$$

where $a_{\mathrm{NN}}$ is the distance to the nearest hopping site, $w_{\mathrm{esc}}$ is the probability for avoiding interface recombination, $x_{0}$ and $U(x))$ are the position and potential along the carrier trajectory, respectively, $\nu_{0}$ is the attempt-to-hop frequency, and $e$ the elementary charge. The inverse localization radius $\kappa$, also known as the tunneling constant, is given by $\kappa=\sqrt{2 m \bar{\varphi}} / \hbar$, with $\bar{\varphi}$ the average barrier height. ${ }^{20}$ The function $\operatorname{Bol}(E)$ is defined as

$$
\operatorname{Bol}(E)=\left\{\begin{array}{ll}
\exp \left(-\frac{E}{k_{B} T}\right), & E>0 \\
1, & E<0
\end{array} .\right.
$$

The carrier escape probability $w_{\text {esc }}$ is given by ${ }^{18}$

$$
w_{\mathrm{esc}}=\frac{\int_{a}^{x_{0}} d x \exp \left(-\frac{e U(x)}{k_{B} T}\right)}{\int_{a}^{\infty} d x \exp \left(-\frac{e U(x)}{k_{B} T}\right)} .
$$

After injection, the current density $j_{\text {bulk }}$ is given by the drift equation $^{21}$

$$
j_{\text {bulk }}(x)=e n(x) \mu[F(x)] F(x),
$$

where $n(x)$ is the local density of mobile carriers and the field-dependent mobility $\mu(F)$, which is characteristic for hopping transport, is given by ${ }^{21}$

$$
\mu(F)=\mu_{0} \exp (\gamma \sqrt{F}) .
$$

Of course, charge conservation demands the equality of $j_{\text {inj }}$ and $j_{\text {bulk }}$ at the injecting contact. Note that, owing to 
electron-hole recombination, $j_{\text {bulk }}$ can decrease along the carrier trajectory. However, as will be shown below, one type of charge carrier generally dominates the current, and thus, this effect can be ignored here.

We note that, in addition to carrier transport via hopping states, the possibility of direct tunneling between the tip and the substrate and vice versa also exists. Following Ref. 20, the tunneling current density $j_{\text {tun }}$ is given by

$$
j_{\text {tun }}=\frac{m e}{2 \pi^{2} \hbar^{3}} \int_{-e V}^{0} d E \int_{-e V}^{E} d W D(W),
$$

where the substrate Fermi level has been taken as the point of zero energy, $W$ is the normal component of the energy, and $m$ is the electron mass. The transmission coefficient $D(W)$ is evaluated in the WKB approximation

$$
D(W)=\exp \left[-2 \sqrt{2 m / \hbar^{2}} \int_{0}^{L} d x[U(x)-W]^{1 / 2}\right] .
$$

For trajectory lengths $L$ of more than a few nanometers, the contribution of $j_{\text {tun }}$ to the total current can be ignored. Note that by using Eqs. (7) and (8), it is implicitly assumed that no resonant states are present in the tunneling barrier.

The potential $U$ is calculated from Poisson's law in cylindrical coordinates $\rho, \Theta$, and $z$ :

$$
\frac{\partial^{2} U}{\partial \rho^{2}}+\frac{1}{\rho} \frac{\partial U}{\partial \rho}+\frac{\partial^{2} U}{\partial z^{2}}=\frac{e}{\varepsilon_{0} \varepsilon_{r}} n_{t o t}(\rho, z),
$$

in which $n_{\text {tot }}$ is the total charge density, and the term containing the angular derivative is neglected because of the assumed symmetry of the problem. Once the potential is known, the carrier trajectories are easily obtained, as they follow the field lines. The effect of the image force on the potential is approximated by adding a correction term to the potential along the field line: ${ }^{20}$

$$
V_{\mathrm{img}}(x)=-\frac{e^{2}}{16 \pi \varepsilon_{0} \varepsilon_{r}} \frac{L}{x(L-x)} .
$$

Here, $L$ is the total length of the field line. In principle, Eq. (10) is only valid for planar surfaces. However, because the range of the image force is limited to at most a few nanometers, we expect that the error induced by this approximation is limited as long as the tip apex radius does not drop below this length scale.

From the potential and field along the field lines, the injected current and hence, the carrier density can be calculated. ${ }^{22}$ It will be clear that Eqs. (9) and (2)-(6) form a self-consistent system because the potential, via the carrier density, depends on the injected current, which in turn depends on the potential. This problem is solved by a simple damped iteration procedure.

Equation (9) is solved numerically by successive overrelaxation, which is derived from the Gauss-Seidel method, on a square grid, with typically $400(z) \times 800(\rho)$ points. ${ }^{23}$ The standard boundary conditions for the electric field are applied at the metal-polymer, metal-vacuum, and polymervacuum interfaces. Therefore, the model can handle arbitrary polymer-layer thicknesses.

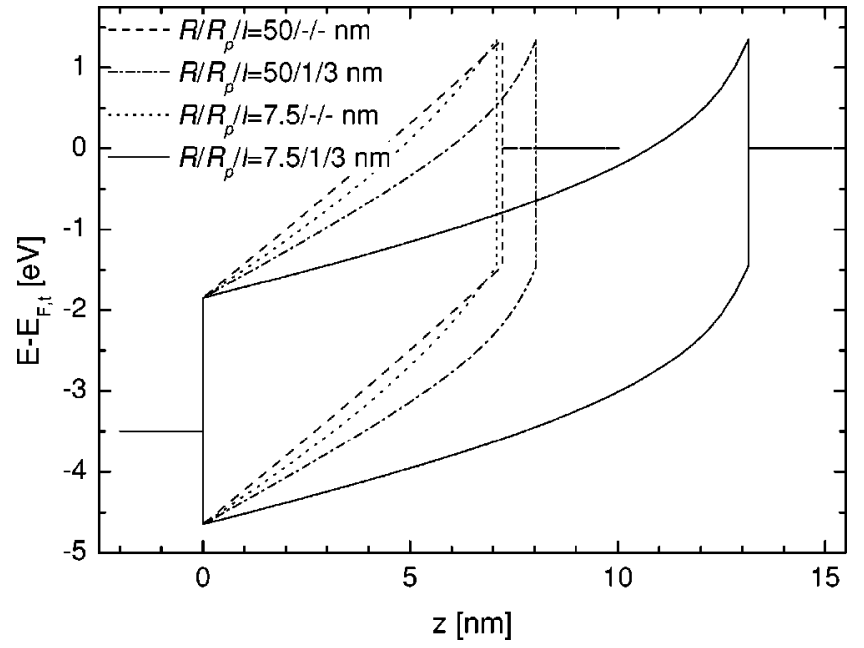

FIG. 2. The potential along the $z$ axis calculated for four different tips, using the typical material parameters for PPV (see the caption of Fig. 3), $E_{\mathrm{g}, \mathrm{sp}}=2.8 \mathrm{eV}, \phi_{\mathrm{e}, \mathrm{t}}=1.35 \mathrm{eV}$, and $\phi_{\mathrm{e}, \mathrm{s}}=1.65 \mathrm{eV}$. The substrate bias and tunneling current are $3.5 \mathrm{~V}$ and $3 \mathrm{pA}$, respectively. The horizontal lines on the left- and right-hand side of the potentials denote the Fermi levels of substrate and tip, respectively.

The equations describing the hopping injection are derived for carrier injection into a semi-infinite slab of organic material. In practice, Eqs. (2) and (4) still hold as long as the length $L$ of the carrier trajectory is more than a few times $a_{\mathrm{NN}}$. However, around $L \approx a_{\mathrm{NN}}$, the model breaks down. In particular, at $L=a_{\mathrm{NN}}, j_{\text {inj }}$ abruptly drops to zero, which is unrealistic because it ignores the variation in $a_{\mathrm{NN}}$ due to spatial disorder in the organic material. Around $L \approx 1 \mathrm{~nm}$, direct tunneling between tip and substrate begins to predominate, and the model becomes valid again. Apart from the range of the tip-substrate distances discussed above, the model presented here has a rather general validity for materials exhibiting hopping-type conductivity. No fundamental limits exist for any dimension, and in principle, any circularly symmetric tip shape can be used. Only when material parameters become position-dependent on a length scale that is comparable to, or smaller than, the tip apex radius, or when a highly irregular tip or substrate is used, is a more elaborate model required.

Our model can be regarded as an extension of the work of Datta et al. ${ }^{24}$ to layers consisting of many molecules. In Ref. 24 it is shown that the electrostatic potential of a single dithiol molecular layer on a gold substrate is not equal to that of the metallic substrate, but lies in between those of the substrate and the tip. However, it will become clear that a model that simply determines the electrostatic potential in the polymer layer by linear interpolation between the substrate and the tip cannot describe our experimental findings.

To illustrate the influence of the tip sharpness, we calculated the tip-sample separation and the potential along $z$ at $\rho=0$, i.e., on the symmetry axis of the tip at $V=3.5 \mathrm{~V}$ and $I=3 \mathrm{pA}$ for four different tips, using the typical material parameters of $p$-phenylenevinylene (PPV), see Fig. 2. The geometry-induced band bending near the tip apex is clearly visible for the tips with $R=7.5 \mathrm{~nm}$, and is strongly enhanced 
by the presence of a parabolic protrusion. As a result, the distance required for $I=3 \mathrm{pA}$ at $V=3.5 \mathrm{~V}$ is always larger for tips with a protrusion, than for those without it. The almost equal tip-sample distance for the tips without a protrusion is due to a trade off between the tip area and geometry effects. Finally, note that for all tips, except the one with $R / R_{p} / l$ $=50 / 0 / 0 \mathrm{~nm}$, the current is carried by electrons that are injected by the tip to more than $99 \%$, notwithstanding the fact that the barrier for hole injection at the substrate $\left(\varphi_{\mathrm{h}, \mathrm{s}}\right)$ is $0.2 \mathrm{eV}$ lower than $\varphi_{\mathrm{e}, \mathrm{t}}$, the electron barrier at the tip. This is the combined result of the tip-induced band bending and the approximately exponential dependence of the injected current on the field at the injecting contact. Although the exact tip-sample distance at which geometry effects become relevant depends on the bias and band alignment, a rule of thumb is that geometry effects become important once the tip-sample separation is on the order of, or larger than, the tip apex radius.

\section{EXPERIMENT}

Two different experimental setups were used. A brief description of the experimental details is given in the following: In the first setup, $z-V$ spectroscopy measurements were performed under UHV conditions (lower $10^{-10}$ mbar range) on the polymers: (1) ladder-type poly(para-phenylene) (Me -LPPP) and (2) poly[p-phenylenevinylene (PPV)]. Both films were prepared under ambient conditions on $\mathrm{Au}(111)$ thin films deposited on mica. The Me-LPPP thin film was prepared by dip coating the substrate in a $0.7 \%$ toluene solution, yielding a film of about $40 \mathrm{~nm}$ thickness. The PPV thin films were prepared by in situ thermal conversion, i.e., under UHV conditions, of a toluene-solvable precursor. ${ }^{25}$ The latter was deposited onto the substrate by spin coating. After conversion, the film was approximately $10 \mathrm{~nm}$ thick. The organic films were inserted into the UHV system through a load lock within a few minutes after the coating of the substrates. The procedure used for coating the substrates resembles that used for making organic light-emitting devices, but our substrate and sample prepration procedures provide cleaner substrate and polymer materials. By curing the PPV prepolymer films in UHV, we eliminated any chemical reaction of the prepolymer with air, ambient contaminants, and residual gases, thus providing conditions for reproducible results. STM-excited electroluminescence measurements of PPV prepared under different ambient conditions show that residual contamination is so important that not only does the luminescence yield improve upon going from preparation under ambient, inert gas, or low vacuum conditions (mbar range) to preparation in the $10^{-7} \mathrm{mbar}$ range, but also an additional improvement is observed when curing the samples under UHV conditions (in the lower $10^{-10}$ mbar range). This reveals differences between PPV samples that are thermally converted under UHV conditions, and samples that are treated in an Ar atmosphere, where reactions with oxygen and water vapor cannot be excluded. The photoluminescence spectrum of the PPV and Me-LPPP polymers closely resembles the results published by other authors. For more experimental details regarding Me-LPPP and PPV, see Refs. 26 and 27, respectively. The $z-V$ spectra were collected using commercially available PtIr tips having a nominal apex radius of $50 \mathrm{~nm}$. Note that each of the spectra of the PPV samples represents the average of several hundred $z-V$ curves collected while scanning the tip over a small, flat area of the substrate surface a few square nanometers in size. ${ }^{6}$ As a result, the averaged spectra may exhibit features whose height is smaller than the diameter of the polymer chains. In particular, some tailing of the $z-V$ curves may result near the charge-injection threshold. For the Me -LPPP samples, the $z-V$ spectra were collected on a single spot without scanning the tip during the voltage sweep. ${ }^{26}$ The tips were cleaned by heating them to $\sim 900^{\circ} \mathrm{C}$ under UHV conditions for 15-30 s. Before data collection, the tips were tested on a separate clean $\mathrm{Au}(111)$ substrate.

In the second setup, ${ }^{28}$ the measurements were done in a $\mathrm{He}$ atmosphere. Here we used Pt tips that were electrochemically etched ${ }^{29}$ from $0.15-\mathrm{nm}$ polycrystalline wire. These tips have a smooth apex with a radius of typically $50 \mathrm{~nm}$, as was confirmed by scanning-electron microscopy. The samples investigated in this setup are spin cast onto a gold electrode layer that is thermally evaporated on a glass substrate. The films are made of poly(2-methoxy-5(3',7'dimethyloctyloxy)-1,4-phenylenevinylene) (MDMO -PPV) which is cast directly from warm $\left(60^{\circ} \mathrm{C}\right)$ chlorobenzene. The typical film thickness is $100 \mathrm{~nm}$. After preparation, the samples are stored and transported under vacuum. While being mounted in the microscope, the samples are exposed to air for about $15 \mathrm{~min}$ before the $\mathrm{He}$ atmosphere is established. $^{30}$

Even at the lowest current densities and highest biases, no meaningful topography is observed on any of the films mentioned above. This is due to the extremely low conductivity of the used materials, which causes the vacuum gap between the tip and the sample to collapse. Because of these observations, no vacuum gap is included in the modeling.

\section{RESULTS}

Figure 3 shows $z-V$ curves from three separate measurements on PPV taken under UHV conditions. The $z-V$ curves were collected by sweeping the bias voltage, while keeping a constant tunneling current of $3 \mathrm{pA}$. Note that in the voltage range of these measurements, the tip has broken through the free surface of the organic thin film, and thus the injection of charge carriers takes place through the potential barrier at the interface between the metal tip and the organic material. It can be seen that at low bias voltage magnitudes, i.e., below $\sim 1.4 \mathrm{~V}$ for negative and below $\sim 0.9 \mathrm{~V}$ for positive tip bias, the tip-substrate separation, $z$, increases with an increasing bias at a rate that is characteristic of the clean $\mathrm{Au}(111)$ surface. This is shown in more detail in the inset, which compares control $z-V$ curves collected on a clean, uncoated $\mathrm{Au}(111)$ surface, with the curves obtained in the presence of a PPV film. We denote the region of coincidence of the two curves as the plateau region of the $z-V$ curve. When the bias voltage exceeds a threshold value, which is different for each tip polarity, the tip-substrate separation increases much more steeply with increasing bias. The characteristic plateau and 


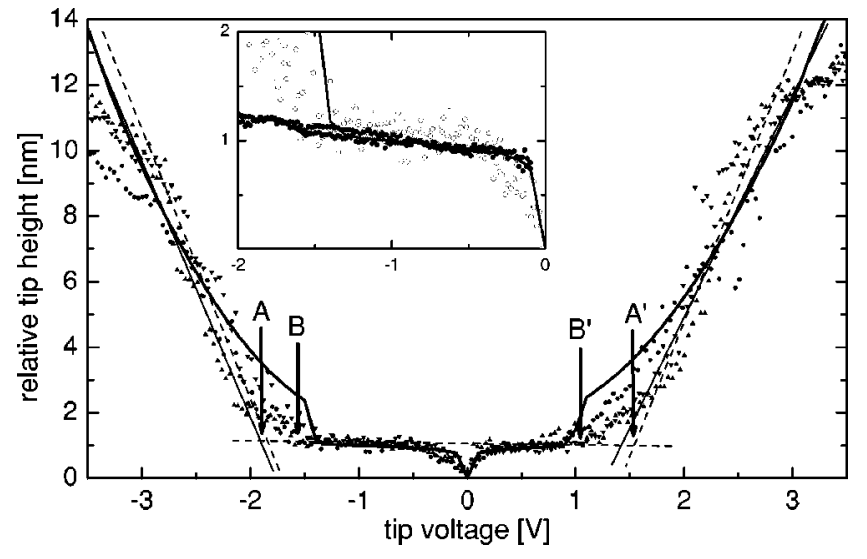

FIG. 3. $z-V$ curves for pure PPV, taken under UHV conditions using a Pt:Ir tip and a current set point of $3 \mathrm{pA}$. The different symbols denote three separate measurements; the thick lines are calculations. The thin dashed and the solid lines extrapolate the high-bias slopes to small $z$ for the measurements and calculations, respectively. The inset shows the $z-V$ curves on pure PPV (open symbols), and as measured on the clean Au (111) substrate (filled symbols), in the low-bias regime. The parameters used in the calculations are $R / R_{\mathrm{p}} / l=7.5 / 1 / 3 \mathrm{~nm}, a_{\mathrm{NN}}=1.1 \mathrm{~nm}, \Gamma=0.11 \mathrm{eV}$, $E_{\mathrm{g}, \mathrm{sp}} / \phi_{\mathrm{e}, \mathrm{t}} / \phi_{\mathrm{e}, \mathrm{s}}=2.8 / 1.35 / 1.65 \mathrm{eV}, \chi_{\mathrm{ts}}=5.4 \mathrm{eV}$.

the threshold behavior have been observed for a series of molecular and conjugated-polymer organic materials. ${ }^{6-9,26}$ Alvarado et al. ${ }^{6}$ have proposed that these thresholds mark the onset of charge-carrier injection into positive (negative) polaronic states, $P^{+}\left(P^{-}\right)$, at an energy $E_{P}^{+}\left(E_{P}^{-}\right)$of the organic material, whereas the width of the plateau corresponds to $E_{\mathrm{g}, \mathrm{sp}}$. Moreover, they argue that a necessary condition for the determination of barrier heights for hole and electron injection at organic/metal interfaces is that the electric field at the tip apex be high enough to induce dominant carrier injection from the tip, with a negligible injection of charges of opposite polarity from the counter electrode. The model calculations reported below show that this requirement of the simple model described in Refs. 6 and 9 is indeed necessary when attempting to determine $\phi_{\mathrm{h}, \mathrm{s}}, \phi_{\mathrm{e}, \mathrm{s}}$, and thus, $E_{\mathrm{g}, \mathrm{sp}}$, from $z-V$ data. This is exemplified by the model calculations performed to fit the data of Fig. 3. The fit is reasonably good for tip-substrate distances larger than one nearest-neighbor intermolecular distance. For shorter distances, discrepancies occur as expected (see Sec. II). Nevertheless, two characteristic features in this intermediate regime are correctly reproduced by the calculation, namely, the points where the plateaus end ( $B$ and $B^{\Re}$ ), and the intersection points ( $A$ and $A^{\Re}$ ), which are defined by the extrapolation of the high-bias curves to the plateau level. For the modeling calculations of Fig. 3, literature values were taken for the material properties. ${ }^{31}$ The single-particle band gap and the molecular orbital level alignment of PPV were taken as fitting parameters. We found that a reasonable fit to the experimental data required a tip apex radius of one to a few nanometers, which indeed causes the tip to be the predominant charge-carrier injector at both polarities. Although the apex radius of the tip is determined in the calculations, the exact tip shape cannot be determined from the modeling. ${ }^{32}$ Apart from determining whether the tip
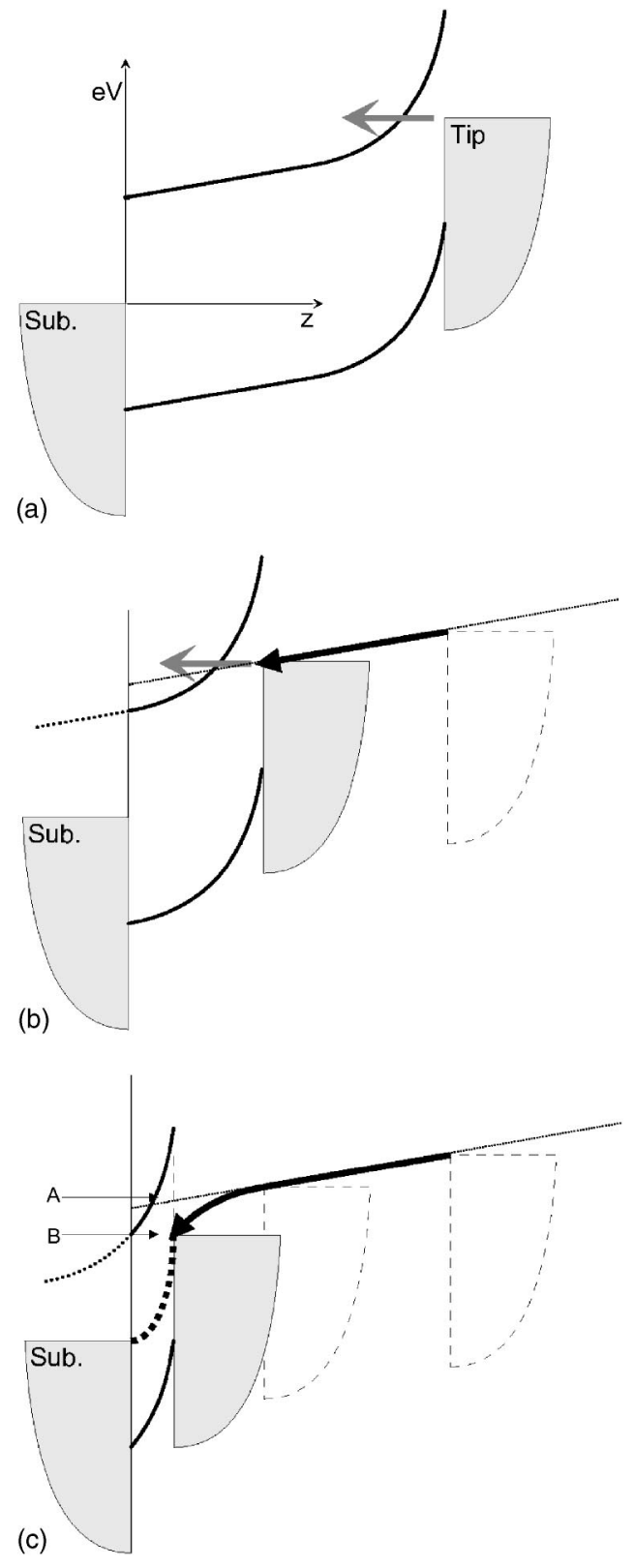

FIG. 4. The schematic band diagrams illustrate the relation between the electrostatic potential and measured $z-V$ curves, for the case in which the (sharp) tip dominates carrier injection. The panels correspond to (a) a large, (b) an intermediate, and (c) a small tipsample separation. The thick solid and dotted lines indicate $z-V$ curves in the hopping injection and tunneling regime, respectively. Note that in this simple approximation the bands only shift, but do not change shape. The gray arrows indicate the predominant injection path.

is the predominant injector, the tip shape affects the high-bias slopes of the $z-V$ curve. Therefore, the geometric tip parameters used in the calculations are only to be regarded as indicative.

A schematic construction of a $z-V$ curve fitting the data in Fig. 3 is shown in Figs. 4(a)-4(c). Three basic conditions need to be fulfilled for this construction. ${ }^{8}$ First, the STM feedback system has to keep the current constant by changing $z$ when $V$ is ramped. Second, the injected current is only 
determined by the potential barrier and field near the chargeinjecting contact. Third, once the potential barrier and field near the injection contact and the current density are known, the remainder of the potential is fixed and can be obtained by a straightforward integration of the Poisson equation. The model presented in Sec. II fulfills these conditions, of course.

In Fig. 4(a), the sharp tip acts as the sole injecting contact because of the strong band bending in the vicinity of its apex. When the magnitude of $V$ is being reduced, the tip, according to the first condition above, has to move forward to keep the injected current constant. According to the second condition, this implies the constancy of the field at the tip, which, because of the third condition, translates into an unchanged potential distribution throughout the entire structure, up to the substrate. Thus, with a changing bias, the tip simply has to follow the fixed potential distribution, as indicated in panel (b). In other words, the tip movement closely reflects the internal potential distribution in the device under operation, which was already shown in Ref. 8 For the situation sketched in Fig. 4, this suggests that the tip motion is approximately linear in $V$ as long as the region of band bending does not extend up to the substrate [see Fig. 4(b)]. At a smaller tip-sample separation, i.e., a lower bias, the $z-V$ characteristic can exhibit a curved region. When the bias drops below a threshold value, $\left|e V_{\mathrm{s}}\right|<\phi_{\mathrm{e}, \mathrm{s}}$, see panel Fig. 4(c), the $P$-level states of the organic material shift above the Fermi level of the tip, and thus the injection of electrons into the organic material is no longer possible. Under these conditions, carrier transport can only take place via direct tunneling between the tip and the sample, and the slope of the $z$ $-V$ curve thus changes abruptly, as indicated by the thick dashed line in Fig. 4. For reversed bias, the tip remains the predominant charge-carrier injector because of its sharp apex, but in this case for positive charge carriers, i.e., holes. The same argumentation can be used to explain the formation of another plateau, starting at $\left|e V_{\mathrm{s}}\right|=\phi_{\mathrm{h}, \mathrm{s}}$ Therefore, the sum of the plateaus in this situation, i.e., for unipolar injection, is $\phi_{\mathrm{e}, \mathrm{s}}+\phi_{\mathrm{h}, \mathrm{s}}$, which is equal to the single-particle band gap $E_{\mathrm{g}, \mathrm{sp}}$. The interpretation of features $A, A^{\Re}$ and $B, B^{\Re}$ in Fig. 3 follows directly by comparison with Fig. 4(c). The latter features are a measure of the single-particle band gap, whereas the differences $A-B$ and $A^{\Re}-B^{\Re}$ may, in part, reflect the geometry-induced band bending in the vicinity of the tip apex.

With the discussion of Fig. 4 in mind, features $B$ and $B^{\Re}$ in Fig. 3 can now be interpreted as the electron and hole barriers at the substrate, $\phi_{\mathrm{e}, \mathrm{s}}$ and $\phi_{\mathrm{h}, \mathrm{s}}$, respectively. This fixes the calculated $z-V$ curve in the low-bias region. However, the distribution of $E_{\mathrm{g}, \mathrm{sp}}$ over the electron- and hole-injection barriers at the tip, $\phi_{\mathrm{e}, \mathrm{t}}$ and $\phi_{\mathrm{h}, \mathrm{t}}$, still needs to be determined. For our injection-limited structure, this distribution follows from the high-bias slopes of the $z-V$ curves. Increasing $\phi_{\mathrm{e}, \mathrm{t}}$ (and therefore decreasing $\phi_{\mathrm{h}, \mathrm{t}}$ ), decreases the high-bias slope at positive $V_{s}$ and simultaneously increases it at negative $V_{s}$. Because the high-bias slope, $d z / d V$ reflects the field $d V / d z$ needed to inject the preset current into the structure, an increase in the injection barrier leads to a decrease of the highbias slope via an increase of the required field.

In general, however, the high-bias slopes of the $z-V$ curves reflect the field needed to obtain the preset current

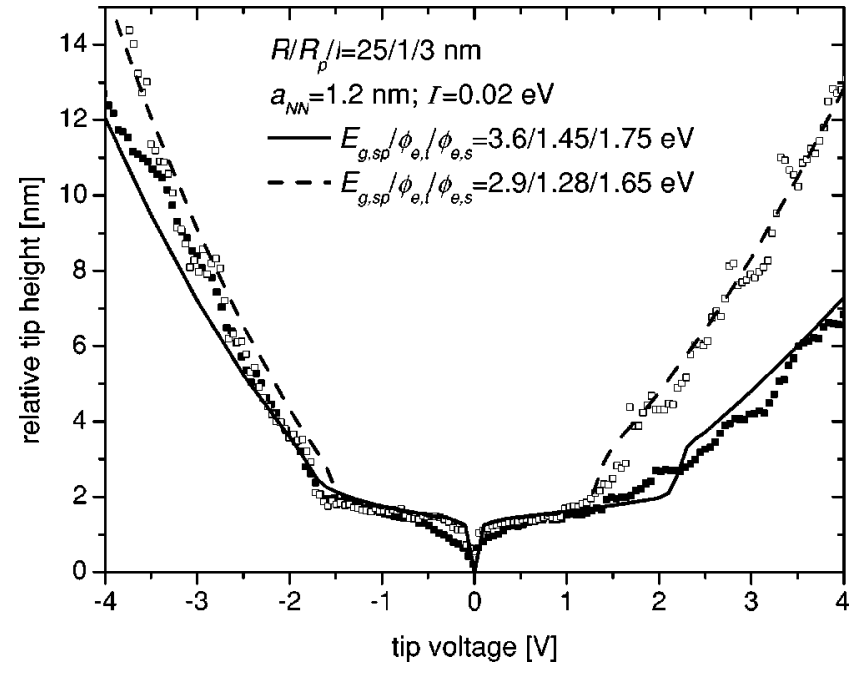

FIG. 5. $z-V$ curves for Me-LPPP taken under UHV conditions with a Pt:Ir tip and a current setpoint of $3 \mathrm{pA}$. The different symbols denote measurements taken with the same tip on different spots of the sample; the lines are calculations to fit the data.

from tip to substrate, and are therefore determined by both the contact resistivity at the tip and the bulk resistivity. In this situation, at least three parameters (electron and hole mobility and the distribution of $E_{\mathrm{g}, \mathrm{sp}}$ over the barriers at the tip, assuming a known tip geometry) have to be determined from two slopes, giving an underdetermined problem. Only when either the bulk or the tip-contact resistivity is dominant, is the problem well posed. ${ }^{33}$

A closer inspection of the calculated $z-V$ curve in Fig. 3 reveals that the gap between points $B$ and $B^{\Re}$ is about $2.6 \mathrm{~V}$, which seems to contradict $E_{\mathrm{g}, \mathrm{sp}}=2.8 \mathrm{eV}$, used in the calculation. The reason for this discrepancy is the Gaussian broadening of the $P^{+}$and $P^{-}$levels of $0.11 \mathrm{eV}$. As the bias at which the tip moves away from the plateau is determined by the energy at which sufficient states in the polymer are available as final states for injection, this bias depends on the level broadening, the temperature, and the required current, amongst other parameters.

The ability to extract the local single-particle gap from $z-V$ spectroscopy can be exploited on spin-cast films of ladder-type Me-LPPP. ${ }^{26,34}$ In this material, spatial variations in $E_{\mathrm{g}, \mathrm{sp}}$ can be expected, either from the formation of aggregates ${ }^{35,36}$ or from differences in orientation of the conjugated backbone with respect to the substrate. ${ }^{37}$ In addition, keto defect sites can occur in the polymer chain during synthesis of the material, ${ }^{38}$ which can also give rise to variations in $E_{\mathrm{g}, \mathrm{sp}}$. Figure 5 shows two $z-V$ curves taken on different spots of the same Me-LPPP film using the same (sharp) Pt: Ir STM tip. The differences in the charge-injection thresholds and slopes at positive tip polarity indicate that in these two spots, the hole barriers at the tip and the substrate differ significantly. For negative tip polarity, however, the similarity of the $z-V$ curves suggests similar electron barriers at both spots. The results yield a difference of about $0.6 \mathrm{eV}$ in $E_{\mathrm{g}, \mathrm{sp}}$, a conclusion that is substantiated by the numerical simulations. A histogram of the distribution of $E_{\mathrm{g}, \mathrm{sp}}$ measured at different positions of the Me-LPPP thin film shows that this 
material contains regions having different band gaps. ${ }^{26}$ This is in contrast to other polymers studied by this technique, for which the histogram reveals a single, inhomogeneously broadened distribution peak.

A comparison of the experiments in Figs. 3 and 5 reveals some differences in the plateau regions, of which the height and shape of the plateaus are the most pronounced. Related to this, the disagreement between the calculation and the experiment at intermediate bias, which is due to the breakdown of the model at $z=a_{\mathrm{NN}}$, is less pronounced for MeLPPP than for PPV. This subject will be further discussed in Sec. V.

The determination of the electronic properties of the organic-metal interface, as shown in Figs. 3-5, requires unipolar injection of charge carriers from the tip. This is achievable by using tips with a very sharp apex, which improves charge injection, as shown in Fig. 2. The condition of unipolar injection, however, does not necessarily hold when a blunt tip is used. This situation can be further aggravated when the difference between $\phi_{\mathrm{e}}$ and $\phi_{\mathrm{h}}$ at either the tip or the substrate interface is large. Consider, for instance, the case depicted schematically in Fig. 6, where the barrier for hole injection at the organic-metal and organic-tip interfaces is much smaller than the barrier for electron injection. Here, the current flowing through the tip-organic-substrate junction would be substrate dominated (hole injection into the organic material) for negative tip polarity and tip dominated for reversed polarity (holes injected from the tip into the organic material). Hence, in this example, the negative-tip-voltage branch of the $z-V$ curve would be a measure of the barrier height for holes at the organic-substrate interface (instead of the desired electron barrier at the organic-substrate interface), while the other branch would reflect the barrier height for holes at the tip-organic interface. Thus the $z-V$ curve would exhibit a plateau that is much narrower than the actual $E_{\mathrm{g}, \mathrm{sp}}$ of the organic material. This is the case in Fig. 7, which shows $z-V$ curves obtained with a Pt tip on MDMO-PPV. Here, no appreciable plateaus are visible in the measured curves.

Despite the relative bluntness of the tip used for these measurements, geometry effects are also important in this situation, as can be concluded from a comparison of the curves calculated with a one-dimensional (1D, dashed lines) and a three-dimensional (3D, solid lines) model, and the experimental data in Fig. 7. Both model curves are calculated using the same material parameters, only the band alignment is varied to reproduce the experimentally observed slopes. Because the device is hole-only at both polarities, the electron barriers and thus, the single-particle band gap, do not influence the calculated curves, and therefore cannot be extracted from the data. Because of the low current density in the experiment, the band bending due to the injected space charges is relatively small, as can be seen from the 1D calculation (dashed and dashed-dotted lines). Geometry effects being absent in the 1D model, the entire curvature results from charging effects. The curvature that is present in both the full 3D model and in the experiment can therefore be attributed to geometry effects. The deviation between the 3D model and experiment is less than the experimental noise, apart from the region below $V_{s}=-6 \mathrm{~V}$, which can be attrib-

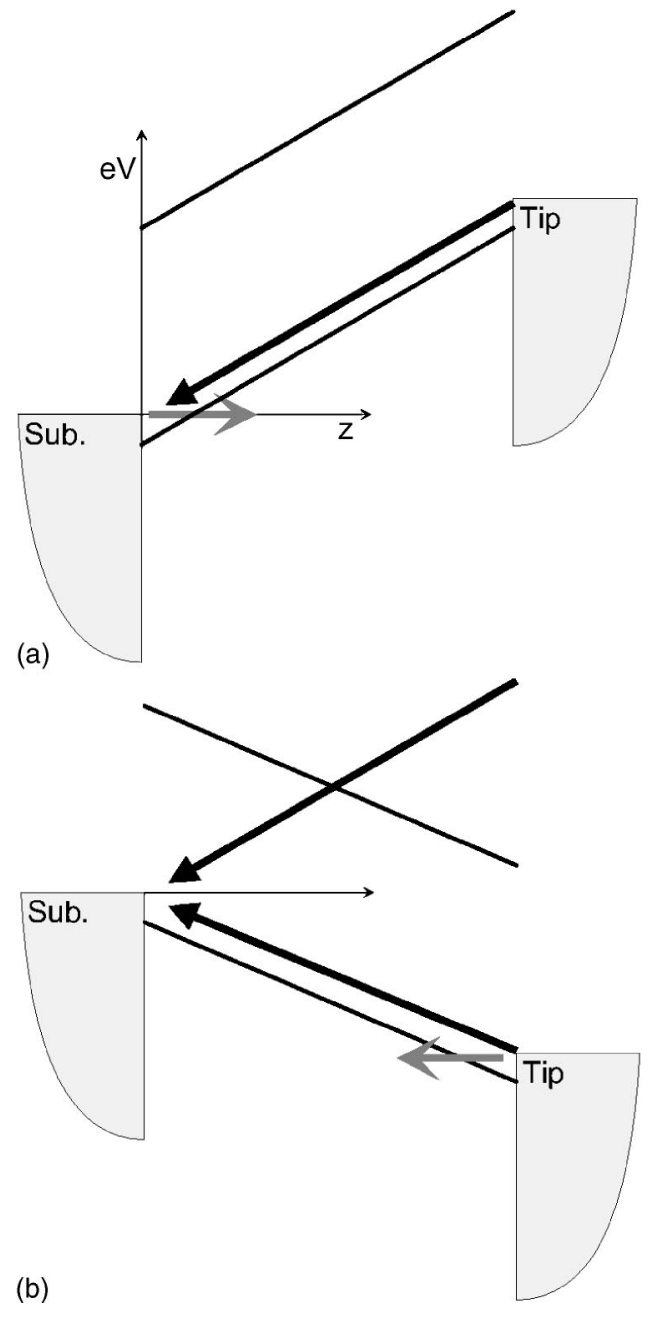

FIG. 6. The schematic band diagrams illustrate the relation between the electrostatic potential and measured $z-V$ curves. In this case, the substrate and the (blunt) tip act as hole injectors. (a) At positive (negative) bias, the substrate (tip) acts as predominant contact. (b) Thick black arrows indicate $z-V$ curves; gray arrows the predominant injection path. Note the absence of significant plateaus in the $z-V$ curve.

uted to an imperfect regulation of the feedback system. ${ }^{39}$ Note that in Ref. 8, a similar nonlinearity in $z-V$ curves was tentatively attributed to the presence of positive and negative charges of an unknown origin near the injecting contacts. Our simulations, however, provide a much simpler explanation for the bended $z-V$ curves taken with a blunt tip.

On the same sample, $I-V$ spectroscopy was also performed (see Fig. 8). With the same material parameters as in Fig. 7 and only minor changes to the band-alignment parameters, the $I-V$ curves could be reproduced by the numerical model. Compared with $z-V$ curves, the effect of geometryinduced band bending on the $I-V$ curves appears to be relatively small, as suggested by the small difference between the $1 \mathrm{D}$ and the 3D models in terms of both the calculated curves and the parameters used. However, when the same parameters are used in the 1D and 3D calculations, substantial differences in the current density are obtained.

The significance of the tip geometry is further addressed in Fig. 9, where calculated $I-V$ curves are shown for four 


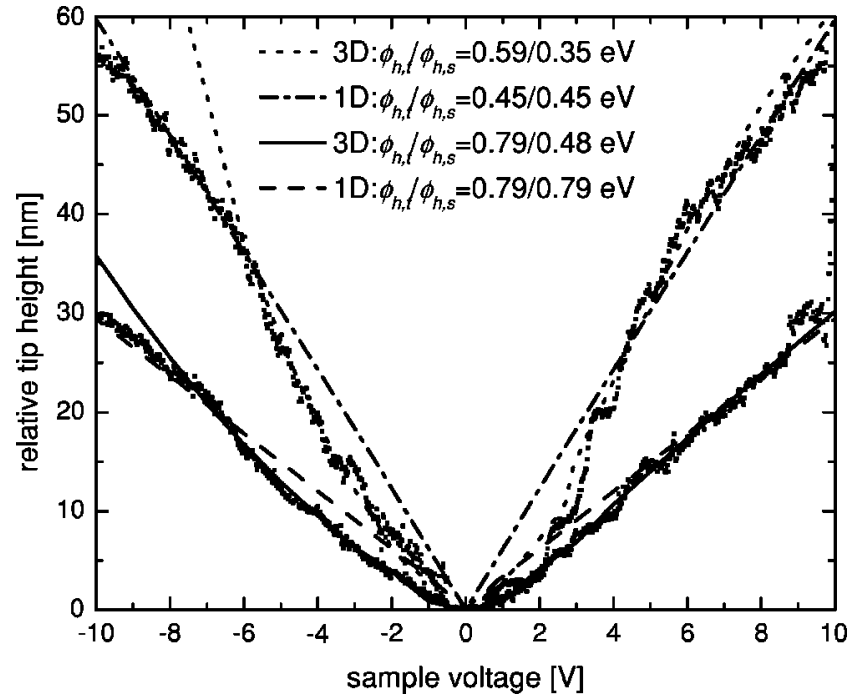

FIG. 7. $z-V$ curves for MDMO-PPV, taken under He gas and using an etched Pt tip. The symbols denote four separate sets of measurements taken on the same sample. The dashed and solid lines are calculations performed with a $1 \mathrm{D}$ and 3D model, respectively. $R=50 \mathrm{~nm}, \mu_{0, \mathrm{ho}}=5 \times 10^{-11} \mathrm{~m}^{2} / \mathrm{V} \mathrm{s}, \gamma=5.4 \times 10^{-11}(\mathrm{~m} / \mathrm{V})^{1 / 2}, \chi_{\mathrm{t}}, \chi_{\mathrm{s}}$ $=5.1 \mathrm{eV}, a_{\mathrm{NN}}=1.2 \mathrm{~nm}$, and $\Gamma=0.11 \mathrm{eV}$.

different situations. A sharp and a blunt tip are considered with either low $(0.3 \mathrm{eV})$ hole-injection barriers for the tip and the substrate, resulting in a space-charge-limited current, or high $(1.0 \mathrm{eV})$ hole-injection barriers, resulting in an injection-limited current. As the single-particle band gap is $2.6 \mathrm{eV}$, the structure is hole-only if the geometry effects are disregarded. Because the injection barriers are equal at the tip and the substrate, the entire asymmetry in the $I-V$ curves is due to geometry effects. As expected, the reduction of the injection-barrier thickness at the tip by geometry-induced field enhancement is most pronounced for the injectionlimited structure. In this case, the sharp tip, despite its

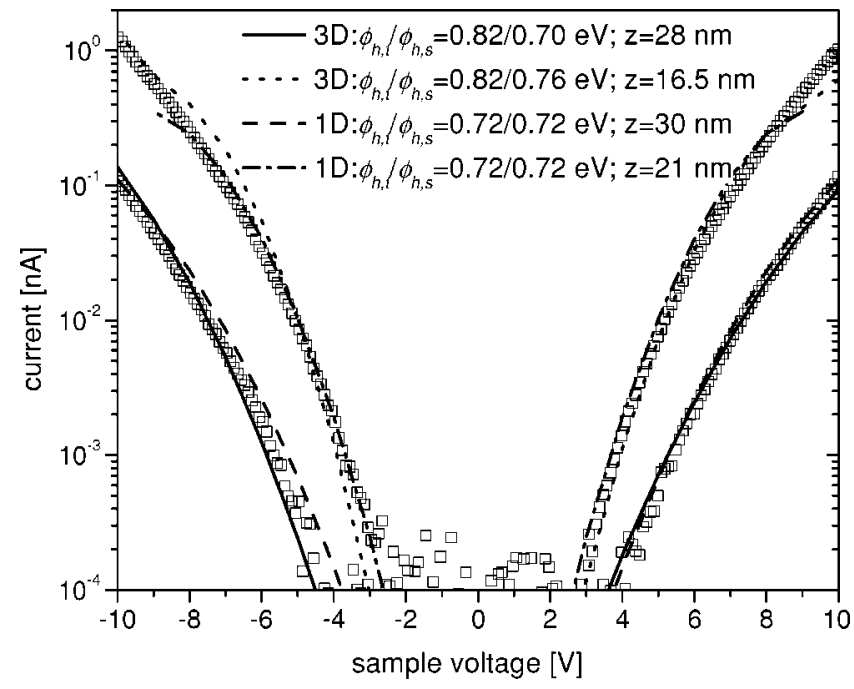

FIG. 8. Current-voltage curves for MDMO-PPV taken under He gas using an etched Pt tip. The STM setpoints are $0.1 \mathrm{nA}$ (squares) and $1.0 \mathrm{nA}$ (circles). $R=100 \mathrm{~nm}, \mu_{0, \mathrm{ho}}=5 \times 10^{-11} \mathrm{~m}^{2} / \mathrm{V} \mathrm{s}, \gamma=5.4$ $\times 10^{-11}(\mathrm{~m} / \mathrm{V})^{1 / 2}, \chi_{\mathrm{t}}, \chi_{\mathrm{s}}=5.1 \mathrm{eV}, a_{\mathrm{NN}}=1.2 \mathrm{~nm}, \Gamma=0.11 \mathrm{eV}$.

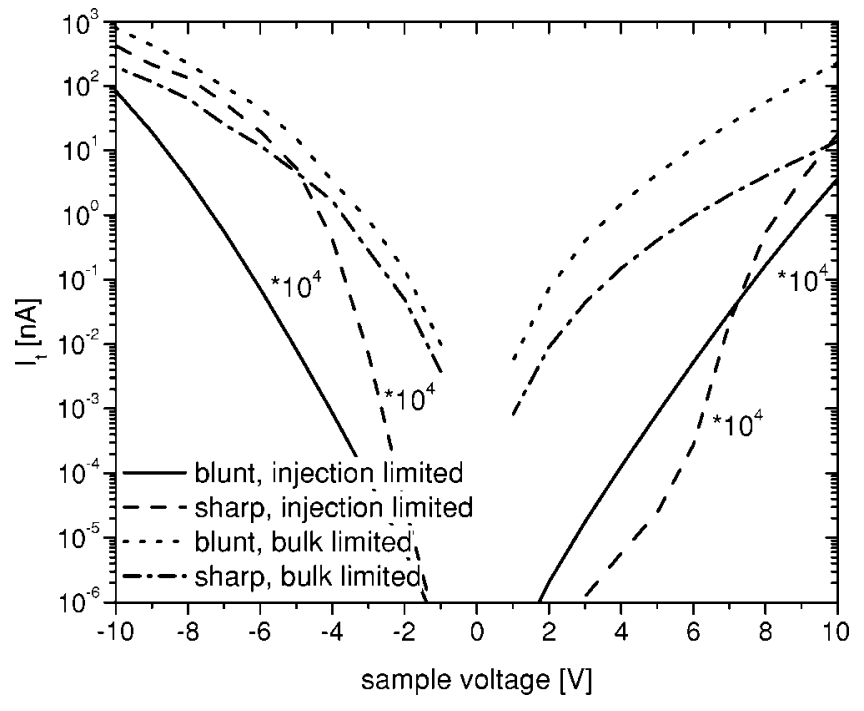

FIG. 9. Calculated $I-V$ curves using the material parameters of MDMO-PPV and a tip-substrate gap $d=25 \mathrm{~nm}$. The following parameters were used: solid line: $R / R_{\mathrm{p}} / l=100 /-/-\mathrm{nm}$, $E_{\mathrm{g}, \mathrm{sp}} / \phi_{\mathrm{e}, \mathrm{t}} / \phi_{\mathrm{e}, \mathrm{s}}=2.6 / 1.6 / 1.6 \mathrm{eV}$; dashed line: $R / R_{\mathrm{p}} / l=25 / 1 / 3 \mathrm{~nm}$, $E_{\mathrm{g}, \mathrm{sp}} / \phi_{\mathrm{e}, \mathrm{t}} / \phi_{\mathrm{e}, \mathrm{s}}=2.6 / 1.6 / 1.6 \mathrm{eV}$; dotted line: $R / R_{\mathrm{p}} / l=100 /-/-\mathrm{nm}$, $E_{\mathrm{g}, \mathrm{sp}} / \phi_{\mathrm{e}, \mathrm{t}} / \phi_{\mathrm{e}, \mathrm{s}}=2.6 / 2.3 / 2.3 \mathrm{eV} ; \quad$ dashed-dotted line: $R / R_{\mathrm{p}} / l$ $=25 / 1 / 3 \mathrm{~nm}, E_{\mathrm{g}, \mathrm{sp}} / \phi_{\mathrm{e}, \mathrm{t}} / \phi_{\mathrm{e}, \mathrm{s}}=2.6 / 2.3 / 2.3 \mathrm{eV}$. The $I-V$ curves for the injection limited structures have been multiplied by a factor of $10^{4}$ for clarity.

smaller injection area, injects about an order of magnitude more (hole) current at a negative sample bias than the blunt tip does. Nevertheless, the asymmetry in the curves for the blunt tip shows that even for blunt tips, geometry effects cannot be ignored in the injection-limited situation. The deviating behavior of the sharp-tip/high-barrier curve at a positive bias is the result of a changeover from hole injection from the substrate below $V_{s} \approx 5 \mathrm{~V}$ to electron injection from the tip above $V_{s} \approx 5 \mathrm{~V}$. In those structures where the current is space-charge (i.e., bulk-) limited, geometry effects are less pronounced, but not absent. The different field distributions at positive and negative bias cause an asymmetry in the $I$ - $V$ curve of the sharp tip of one order of magnitude at the highest bias. For the blunt tip, the asymmetry in the same situation is only a factor two. The fact that the current for the sharp tip is lower than that for the blunt tip is due to the smaller contact area of the sharp tip.

\section{DISCUSSION}

We now discuss the determination of the hole- and electron-injection barriers, the single-particle energy gap, and the exciton binding energy as derived from our $z-V$ spectroscopy data, and compare the results with those obtained by other research groups using different experimental techniques. From the data, shown in Fig. 3, for PPV/Au(111), we obtain the barrier for electron injection $\phi_{\mathrm{e}, \mathrm{s}}$ $=2.8 \pm 0.1 \mathrm{eV}$, and for holes $\phi_{\mathrm{h}, \mathrm{s}}=1.15 \pm 0.1 \mathrm{eV}$. From this we obtain $E_{\mathrm{g}, \mathrm{sp}}=2.8 \pm 0.1 \mathrm{eV}$, which is within the range of that reported in a previous publication ${ }^{27}$ and comparable to the value of about $3 \mathrm{eV}$ obtained by STM-based spectros- 
copy measurements on PPV deposited on GaAs. ${ }^{40}$ One could compare this result with the predictions of the common vacuum level $(\mathrm{CVL})$ rule, which assumes that $\phi_{\mathrm{h}, \mathrm{s}}$ equals the difference between the ionization potential (IP) of the organic material and the work function of the metal. This method, however, is unreliable because the adsorbed organic material can modify the metal surface dipole, which in turn shifts the alignment of the electronic levels of the organic material with respect to the ideal, unperturbed, case. ${ }^{41-43}$ This effect can occur even when the adsorbed molecules have no effective dipole moment. ${ }^{42}$

The electron barrier compares well with values obtained by means of internal photoemission (IPE) on PPV/Au (Ref. 44) and dialkoxy-PPV/Au (Ref. 45) interfaces. There is also good agreement with the value $\phi_{\mathrm{e}, \mathrm{s}}=1.23 \mathrm{eV}$ obtained by Roman et al. ${ }^{46}$ by means of Fowler-Nordheim tunneling (FNt). However, other equally relevant reports, that are at variance with the above results, exist. A value of $\phi_{\mathrm{e}, \mathrm{s}}$ $=2.15 \mathrm{eV}$ (from which $\phi_{\mathrm{h}, \mathrm{s}}=0.3$ is deduced) was obtained by Campbell et $a .^{47}$ by means of IPE on poly[2-methoxy, 5-(2'-ethyl-hexoxyl)-1,4-phenylene vinylene] (MEH-PPV) deposited on Au. Similarly, Parker ${ }^{48}$ finds $\phi_{\mathrm{h}, \mathrm{s}} \approx 0.2 \mathrm{eV}$ by means of FNt on MEH-PPV/Au. Finally, variations of the injection barrier for electrons have also been detected by electroluminescence excited by charge carriers injected from the tip of an STM, ${ }^{7}$ where values of $\phi_{\mathrm{e}, \mathrm{s}}$ from 0.85 to about $2 \mathrm{eV}$ are found for PPV/Au. Clearly, the results differ substantially, even when the same experimental technique was used. The results obtained by different laboratories suggest, however, that they can be grouped as follows: (i) The distribution of energy barriers is such that the Fermi level of the metal substrate is located near the center of the polymer's energy gap, as is the case for this work, Figs. 3 and 5, and for Refs. 44-46. (ii) The Fermi level is located within a few $100 \mathrm{meV}$ above the HOMO (highest occupied molecular orbital) states. ${ }^{8,47,48}$ The reason for these diverging results may lay in the presence of extraneous material at the metal/ organic interface, which can modify the metal/organic interface by either introducing a dipole layer and/or affecting the dipole layer at the interface. Thus, for instance, Ettedegui et $a l .^{49}$ find that the barrier properties depend on the details of the metal/organic surface preparation. In addition, a study of the influence of residual gases adsorbed on a $\mathrm{Au}$ surface reveals that exposure of the substrate to ambient conditions can cause the electronic level alignment of PPV oligomers to shift by about $1 \mathrm{eV}$, relative to the clean Au surface. ${ }^{50}$ It is also known that hydrocarbon absorption on the Au surface can induce a reduction of the Au work function by as much as $1 \mathrm{eV} .{ }^{51}$ The main unknowns are, hence, (i) the nature of the foreign material that might be adsorbed on the substrate and (ii) residual adsorbates stemming from the solvent used in the PPV precursor. Moreover, the conditions, e.g., UHV or in an Ar atmosphere, ${ }^{49}$ under which thermal conversion of the precursor polymer took place, can also influence the interface.

We now turn to the determination of the singlet exciton binding energy. The magnitude of $E_{\mathrm{b}}$ for PPV has been a highly controversial topic. Experimental studies yield values that differ by more than one order of magnitude, see below, and a full consensus has not yet been reached.
$E_{\mathrm{b}}$ is defined as the energy difference between the singleparticle band gap (polaronic band gap) and the optical absorption edge of the bulk material

$$
E_{\mathrm{b}}=E_{\mathrm{g}, \mathrm{sp}}-E_{\mathrm{a}} .
$$

This definition holds unambiguously in the long conjugated-chain limit. ${ }^{52}$ However, for the typical conjugation lengths, $L_{\mathrm{c}}=6-10$ monomers, ${ }^{53-56}$ which characterize bulk PPV, $E_{\mathrm{g}, \mathrm{sp}}$ and $E_{\mathrm{a}}$ are functions of $L_{\mathrm{c}}$. These functions converge to within a few $\mathrm{meV}$ of the limiting values for the infinite chain only for $L_{\mathrm{c}}>15$ monomers. ${ }^{57-59}$ If we assume an average conjugation length of 6-10 monomers, the value of $E_{\mathrm{b}}$ obtained with Eq. (11) is overestimated by about $80 \mathrm{meV}$. Note also that the exciton binding energy depends on $L_{\mathrm{c}}$, (see, for instance Refs. 59 and 60), and on the chainpacking density. ${ }^{61}$ It is with these precautions in mind that we make an estimate of the exciton binding energy from our experimental data. Thus, with $E_{\mathrm{a}}=2.4 \mathrm{eV}$ and $E_{\mathrm{g}, \mathrm{sp}}=2.8 \mathrm{eV}$ estimated from the modeling calculations to fit the data, we obtain $E_{\mathrm{b}}=0.4 \pm 0.1 \mathrm{eV}$, which compares with the previously estimated value of $0.48 \pm 0.14 \mathrm{eV}$. $^{6}$

This result compares well with IPE results on MEHPPV of Campbell et al. ${ }^{47}$ from which $E_{\mathrm{b}}=0.35 \mathrm{eV}$ is obtained. ${ }^{27,52}$ Similarly, a value of $E_{\mathrm{b}}=0.36 \mathrm{eV}$ was reported for an alkoxy-substituted PPV copolymer. ${ }^{6}$ Note that $E_{\mathrm{b}}$ of PPV is expected to be slightly higher than that of alkoxyPPV compounds because of their higher polarizability, which reduces the Coulomb energy contribution to $E_{\mathrm{b}} \cdot{ }^{27,62,63}$ Our results are also in good agreement with values obtained by other research groups, which report values of $>0.25$ (Ref. 64), 0.3 (Ref. 65), and $0.4 \mathrm{eV}$ (Refs. 66 and 67). We note, however, that $E_{\mathrm{b}}$ values as low as $\sim 60 \mathrm{meV} \mathrm{eV},{ }^{68}$ as well as relatively high values, for instance 0.7 (Ref. 69), 0.8 (Ref. 64), >0.8 (Ref. 70), 0.9 (Ref. 71), and 1.1 (Ref. 72), have been reported. Our results are also in reasonably good agreement with theoretical studies, which yield $E_{\mathrm{b}}$ values of $0.2-0.3 \mathrm{eV}$ for PPV in crystal packing, ${ }^{61} 0.54 \mathrm{eV}$ for a single chain embedded in a dielectric medium simulating bulk material, ${ }^{73} 0.4 \mathrm{eV}$ from an effective mass calculation, ${ }^{74}$ and $0.3 \mathrm{eV}$ from a Monte Carlo simulation. ${ }^{66}$ For an isolated PPV chain, calculations in the literature show a tendency towards higher values of $E_{\mathrm{b}}$ : 0.3 (Ref. 60), 0.4 (Ref. 75), 0.7 (Ref. 59), 0.6-0.7 (Ref. 62), and $0.9 \mathrm{eV} .71,76$

Let us now turn to Me-LPPP, where, as mentioned above, regions having distinctly different values of $E_{\mathrm{g}, \mathrm{sp}}$ are found. The distribution of $E_{\mathrm{g}, \mathrm{sp}}$ in Me-LPP reflects variations of the material properties as well as of the injection threshold at different locations on the sample. The regions having the smallest $E_{\mathrm{g}, \mathrm{sp}}$ have been interpreted as aggregate domains, and a detailed discussion of this point was given in Ref. 26. From the regions of the sample corresponding to the intrinsic, disordered polymer chains, one obtains $\phi_{\mathrm{e}, \mathrm{s}}$ $=1.3 \pm 0.2 \mathrm{eV}$ and the lowest $\phi_{\mathrm{h}, \mathrm{s}}=1.8 \pm 0.15 \mathrm{eV}$ for a $\mathrm{Au}(111)$ electrode, from which $E_{\mathrm{g}, \mathrm{sp}}=3.1 \pm 0.09 \mathrm{eV}$ is deduced. The histogram of the data reveals a second peak at a higher energy, namely, $E_{\mathrm{g}, \mathrm{sp}}=3.45 \pm 0.13 \mathrm{eV} .{ }^{26} \mathrm{We}$ note that the widths of these two peaks compare reasonably well with the peak widths of the vibronic transitions of the photoluminescence and absorption spectra of this material. The two 


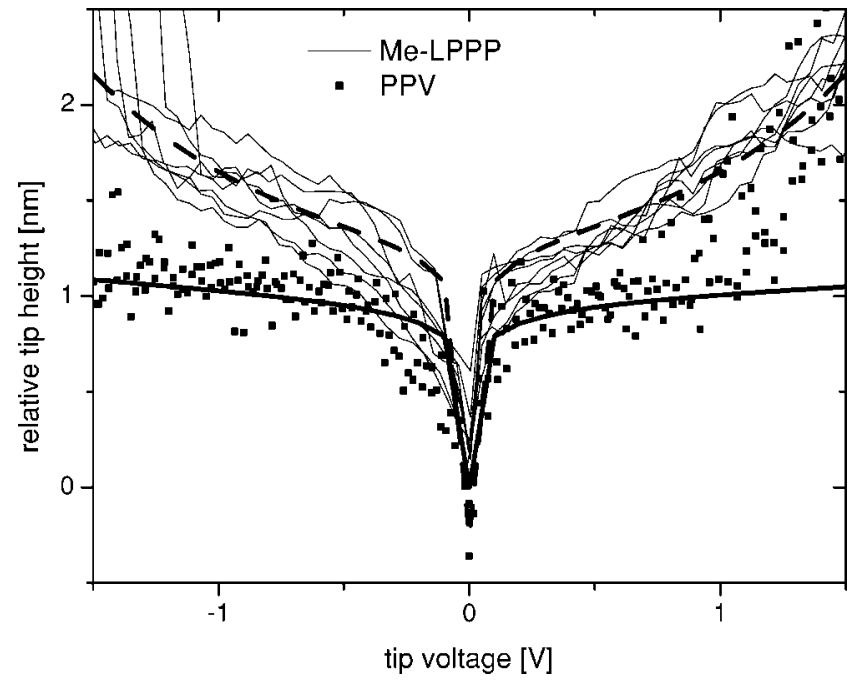

FIG. 10. A closeup of the low-bias region of several $z-V$ curves taken on PPV (symbols) and Me-LPP (thin lines) taken under UHV conditions with a Pt:Ir tip. The thick lines are calculations, assuming tunneling through vacuum (solid line, tunneling barrier height: $5.4 \mathrm{eV}$ ) and nonresonant polymer states (dashed line, tunneling barrier height: $1.5 \mathrm{eV}$ ). In both cases, the dielectric constant of the polymer is used. All parameters are the same as in Figs. 3 and 5.

peaks are much narrower than those of PPV, alkoxy-PPVs, and polyfluorene studied with the STM-based $z-V$ spectroscopy technique. This is in accordance with the weak inhomogeneous broadening of the absorption and the photoluminescence spectra of Me-LPPP as compared with those of the other conjugated polymers. From the above data and by taking $E_{\mathrm{a}}=2.65 \mathrm{eV}$, we deduce binding energies of $0.45 \pm 0.09$ and $0.8 \pm 0.13 \mathrm{eV}$, respectively. This is not necessarily proof that two different types of singlet excitons exist in $\mathrm{Me}$ -LPPP because the optical band gaps corresponding to each $E_{\mathrm{g}, \mathrm{sp}}$ of the sample is not known. The data, however, is not in disagreement with the results of electron energy loss measurements of Knupfer et al. ${ }^{77}$ that indicate the existence of a strongly localized and a delocalized singlet exciton near the absorption onset. Our results for $E_{\mathrm{b}}$ compare reasonably well with the value of $0.6 \mathrm{eV}$ obtained by Wohlgennant et al. using a photomodulation technique. ${ }^{78}$ A study of the field dependence of photogeneration of charge carriers yields $E_{\mathrm{b}}$ $=0.35 \mathrm{eV}^{.41}$

As mentioned in Sec. IV, marked differences exist between the $z$ - $V$ curves of Me-LPPP and PPV in the low-bias regime. Figure 10 shows a closeup of this region. Although the tip height is expressed on a relative scale, i.e., the substrate position cannot be determined independently and therefore has to be estimated from the $z-V$ curve, the dependence of the tip height on the bias suggests a different functional shape for the two polymers. Compared with the PPV curve, the Me-LPPP $z-V$ curve increases more steeply with bias. A likely explanation for these differences is that the barrier experienced by the tunneling electrons differs in the two cases. The calculated curves shown in Fig. 10 use the vacuum level as a barrier for tunneling through PPV, and the $E_{P}^{-}$level as a barrier for tunneling through Me-LPPP. The latter choice implies that nonresonant tunneling takes place
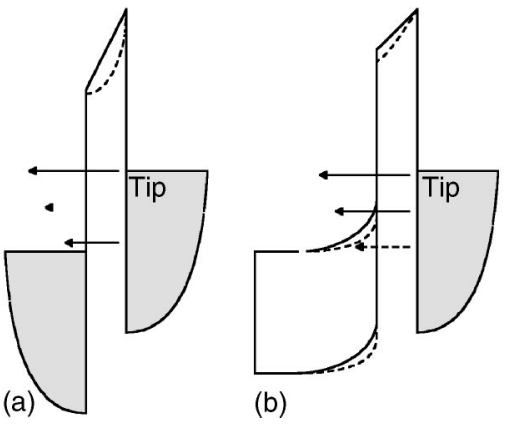

(b)

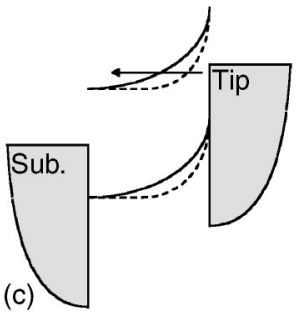

FIG. 11. Schematic energy diagrams for electron injection from a metallic STM tip into (a) a metal, (b) an inorganic semiconductor, and (c) an organic semiconductor. The solid and dashed lines indicate the energy levels in the absence and presence of geometryinduced band bending, respectively. The shaded regions denote metal electrodes.

through states in the polymer. With this choice of tunneling barriers, the experimental curves are reproduced quite accurately by the model. The marked difference is most likely due to a slightly different measurement procedure used for PPV, in which the penetrating STM tip is actually scanning the sample, whereas the tip is kept at a fixed lateral position in the case of Me-LPPP. The former method is, by far, more likely to result in a removal of polymer material from the gap region when the gap narrows.

Regarding our results on MDMO-PPV, we note a remarkable difference in terms of the energy-level alignment at the substrate to our results on unsubstituted PPV. Recalling the above-proposed grouping of literature values for the alignment of the Au Fermi level to the PPV HOMO level, the former falls into the second category (see Fig. 7), whereas the latter seems to belong to the first category (see Fig. 3). Considering the large differences in the preparation conditions of substrates and films used for these two samples, however, this should not come as a surprise. Differences in both the intimacy of the contact between metal and PPV, and in adsorbates on the Au surface are likely to occur, and cause the observed difference in energy-level alignment, as discussed extensively at the beginning of this section.

Finally, we wish to address the question of why geometry effects on the tunneling current are much more important for injection into organic semiconductors than, for example, for injection into metals or inorganic semiconductors. The key parameter here is the field penetration into the sample, as illustrated in Fig. 11. In conventional STM operation on metals, the total applied bias drops over the vacuum barrier [see Fig. 11(a)]. Consequently, geometry-induced band bending affects only the field in the vacuum barrier, but not its height and thickness, resulting in only a minor change in the injected current. Under normal experimental conditions, a part of the field penetrates into an inorganic semiconductor [see Fig. 11(b)], but the majority of the applied bias drops in the vacuum gap because of the large difference in the dielectric constants of the (inorganic) semiconductor and the vacuum. ${ }^{79}$ Geometry effects, therefore, affect the shape of the vacuum barrier and the height of the surface barrier. The latter results in a change in the apparent band gap in a 
constant-height $I-V$ curve. On the other hand, as long as the Fermi level of the tip is higher than the surface barrier, the effect on the tunnel current is limited, as the majority of the current is carried by electrons emitted at the Fermi level of the tip. Based on these arguments, geometry effects on carrier injection are expected to be stronger for inorganic semiconductors than for metallic materials, in agreement with the existing literature. ${ }^{4,3}$ When electrons are injected into the film of an organic semiconductor, the final state of the tunneling process generally lies below, rather than at, the sample surface [see Fig. 11(c)]. This can either be the result of the total absence of a vacuum gap, as is the case in the experiments discussed in this paper, or of a relatively small potential drop across the vacuum gap due to the low dielectric constants of typical organic materials. ${ }^{79}$ In either case, the field at the tip apex is directly linked to the distance the carrier needs to tunnel, and hence to the injection probability that determines the injected current. Owing to the exponential dependence of the tunnel probability on the tunnel distance, geometry effects play a crucial role in carrier injection, as has been shown in this paper.

Of course, the above discussion is far from complete, and situations are conceivable in which geometry effects play a large role in STM injection into inorganic materials-for example, when the Fermi level of the tip aligns with the surface barrier of an inorganic semiconductor, ${ }^{20,80}$ - or they can be irrelevant to injection into organic materials. Nevertheless, the qualitative arguments given here should hold for most practical situations.

\section{CONCLUSION}

We have performed a combined experimental and numerical study on STM-based spectroscopy on conjugated polymers. We show that, because of the sharpness of STM tips, a meaningful interpretation of both current-voltage $(I-V)$ and tip height-voltage $(z-V)$ curves requires the threedimensional nature of the system to be taken into consideration. This holds for injection- as well as for bulk-limited systems. Only when the tip apex radius is much larger than the tip-substrate gap, can reasonable parameters be obtained from a one-dimensional analysis. In all other cases, geometry effects on the carrier injection and transport can alter the device current itself, as well as the balance between the minority and the majority currents by more than an order of magnitude, compared with the one-dimensional situation. Thus, by using a very sharp tip it is possible to make a nanometer-sized device in which the predominant current is of the minority type.

In particular, when a sufficiently sharp tip is used, the single-particle band gap and the band alignment at both electrodes can be obtained. Consequently, the much debated exciton-binding energy can be obtained by subtraction of the optical band gap from the single-particle band gap. The modeling results presented in this report lay a formal theoretical basis to $z-V$ spectroscopy, which is shown to be a powerful technique for probing the electronic structure of organic material interfaces with an electrode. Although we have discussed the particular case of organic/metal interfaces, the $z$ $-V$ spectroscopy technique can also be applicable to organic/organic ${ }^{81}$ and organic/inorganic interfaces.

\section{ACKNOWLEDGMENTS}

We gratefully acknowledge J. K. J. Van Duren for supplying the MDMO-PPV samples and for stimulating discussions, and R. H. I. Keiboams and D. Vanderzande for providing the PPV precursor. Part of the research of M.K. has been made possible by a fellowship of the Royal Netherlands Academy of Arts and Sciences. One of us (S.F.A.) acknowledges W. Riess for his support.
${ }^{1}$ G. J. de Raad, P. M. Koenraad, and J. H. Wolter, J. Vac. Sci. Technol. B 17, 1946 (1999).

${ }^{2}$ O. Albrektsen, H. W. M. Salemink, K. A. Morch, and A. R. Thölen, J. Vac. Sci. Technol. B 12, 3187 (1994).

${ }^{3}$ R. M. Feenstra, J. A. Stroscio, and A. P. Fein, Surf. Sci. 181, 295 (1987).

${ }^{4}$ Th. Laloyaux, I. Derycke, J.-P. Vigneron, Ph. Lambin, and A. A. Lucas, Phys. Rev. B 47, 7508 (1993).

${ }^{5}$ G. I. Márk, L. P. Biró, J. Gyulai, P. A. Thiry, A. A. Lucas, and Ph. Lambin, Phys. Rev. B 62, 2797 (2000).

${ }^{6}$ S. F. Alvarado, P. F. Seidler, D. G. Lidzey, and D. D. C. Bradley, Phys. Rev. Lett. 81, 1082 (1998).

${ }^{7}$ S. F. Alvarado, "STM-Excited Electroluminescence and Spectroscopy of Conjugated Polymers," in Conjugated Polymers and Molecular Interfaces, Science and Technology and Optoelectronic Applications, edited by W. R. Salaneck, K. Seki, A. Kahn, and J.-J. Pireaux (Dekker, New York, 2001), p. 473.

${ }^{8}$ M. Kemerink, P. Offermans, P. M. Koenraad, J. K. J. van Duren, R. A. J. Janssen, H. W. M. Salemink, and J. H. Wolter, Phys.
Rev. Lett. 88, 096803 (2002).

${ }^{9}$ S. F. Alvarado, L. Rossi, P. Müller, P. F. Seidler, and W. Reiss, IBM J. Res. Dev. 45, 89 (2001); www.research.ibm.com/ journal/rd/451/alvarado.pdf

${ }^{10}$ H. Sirringhaus, P. J. Brown, R. H. Friend, M. M. Nielsen, K. Bechaard, B. M. W. Langeveld-Voss, A. J. H. Spiering, R. A. J. Janssen, E. W. Meijer, P. Herwig, and D. M. de Leeuw, Nature (London) 401, 685 (1999).

${ }^{11}$ H. C. F. Martens, O. Hilt, H. B. Brom, P. W. M. Blom, and J. N. Huiberts, Phys. Rev. Lett. 87, 086601 (2001).

${ }^{12}$ P.-K. Wei, Y.-F. Lin, W. Fann, Y.-Z. Lee, and S.-A. Chen, Phys. Rev. B 63, 045417 (2001).

${ }^{13}$ C. Schiller, A. Koomans, T. van Rooy, C. Schönenberger, and H. Elswijk, Surf. Sci. Lett. 339, L925 (1995).

${ }^{14}$ W. A. Hofer, A. J. Fisher, R. A. Wolkow, and P. Grütter, Phys. Rev. Lett. 87, 236104 (2001).

${ }^{15}$ H. Bässler, Phys. Status Solidi B 175, 15 (1993).

${ }^{16}$ Z. G. Yu, D. L. Smith, A. Saxena, R. L. Martin, and A. R. Bishop, Phys. Rev. Lett. 84, 721 (2000). 
${ }^{17}$ E. M. Conwell and M. W. Wu, Appl. Phys. Lett. 70, 1867 (1997).

18 V. I. Arkhipov, E. V. Emelianova, Y. H. Tak, and H. Bässler, J. Appl. Phys. 84, 848 (1998); V. I. Arkhipov, U. Wolf, and H. Bässler, Phys. Rev. B 59, 7514 (1999).

${ }^{19}$ T. van Woudenbergh, P. W. M. Blom, M. C. J. M. Vissenberg, and J. N. Huiberts, Appl. Phys. Lett. 79, 1697 (2001).

${ }^{20}$ R. M. Feenstra and J. A. Stroscio, J. Vac. Sci. Technol. B 5, 923 (1987).

${ }^{21}$ P. W. M. Blom and M. C. J. M. Vissenberg, Mater. Sci. Eng., R. 27, 53 (2000).

${ }^{22}$ Because of the divergence of the field lines, $j_{\text {bulk }}$ decreases when going from tip to sample. In addition, the contribution of the tunneling current to the space charge is ignored because compared with the transition time associated with the drift process, the tunneling time is negligible.

${ }^{23}$ K. J. Binns and P. J. Lawrenson, Analysis and Computation of Electric and Magnetic Field Problems, 2nd ed. (Pergamon, Oxford, New York, 1973).

${ }^{24}$ S. Datta, W. Tian, S. Hong, R. Reifenberger, J. I. Henderson, and C. P. Kubiak, Phys. Rev. Lett. 79, 2530 (1997).

25 L. Louwet, D. J. Vanderzande, and J. M. Gelan, Macromolecules 28, 1330 (1998); D. J. Vanderzande, A. C. Issaris, M. J. van der Borght, A. J. van Breemen, M. M. de Kok, and J. M. Gelan, Macromol. Symp. 125, 189 (1997); A. C. Issaris, D. J. Vanderzande, and J. M. Gelan, Polymer 38, 2571 (1997).

${ }^{26}$ S. F. Alvarado, H. Bässler, U. Scherf, J.-W. van der Horst, P. A. Bobbert, and M. A. J. Michels, Adv. Funct. Mater. 12, 117 (2002).

${ }^{27}$ L. Rossi, S. F. Alvarado, W. Riess, S. Schrader, D. G. Lidzey, and D. D. C. Bradley, Synth. Met. 111-112, 527 (2000).

${ }^{28}$ M. Kemerink, J. W. Gerritsen, J. G. H. Hermsen, P. M. Koenraad, H. van Kempen, and J. H. Wolter, Rev. Sci. Instrum. 72, 132 (2001).

${ }^{29}$ L. Libioulle, Y. Houbion, and J.-M. Gilles, Rev. Sci. Instrum. 66, 97 (1995).

${ }^{30}$ On actual organic light-emitting devices using these types of films as an active layer, no measurable degradation of the operation characteristics is observed for exposure times in this range. Therefore, strong, progressive contamination due to the exposure to ambient conditions can be excluded. However, a slight adsorption of extraneous adsorbates cannot be fully excluded.

${ }^{31}$ The parameters for transport in and hopping injection into PPV were taken from Refs. 21 and 19, respectively. A slightly lower value for $a_{\mathrm{NN}}(2.2 \mathrm{~nm}$ instead of $2.4 \mathrm{~nm})$ was used to account for the smaller unit-cell size of pure PPV compared with the substituted PPV of Ref. 19. Note that the calculated curves in Figs. 3 and 5 are extremely insensitive to the values of $\mu$ and $\gamma$, as the transport is entirely injection limited. The numerical values used in the calculations were those used for MDMO-PPV (as given in Figs. 7 and 8, and their captions).

${ }^{32}$ In the fitting procedure, the tip shape was kept fixed while the gap and the alignment were varied freely. If no satisfactory fit could be obtained, the procedure was repeated with a different tip shape.

${ }^{33}$ The problem may appear overdetermined in the case of injectionlimited structures, but the high-bias slopes depend on the tip shape as well. As such, the reproduction of both slopes by only adjusting the distribution of the single-particle gap over the electron and hole barriers at the tip forms a qualitative confirmation of the tip shape assumed.
${ }^{34}$ D. Hertel, U. Scherf, and H. Bässler, Adv. Mater. (Weinheim, Ger.) 10, 1119 (1998).

${ }^{35}$ R. F. Mahrt, T. Pauck, U. Lemmer, I. Siegner, M. Hopmeier, R. Hennig, H. Bässler, E. O. Göbel, P. Haring Bolivar, G. Wegmann, H. Kury, U. Scherf, and K. Müllen, Phys. Rev. B 54, 1759 (1996).

${ }^{36}$ S. Barth, H. Bässler, U. Scherf, and K. Müllen, Chem. Phys. Lett. 288, 147 (1998).

${ }^{37}$ J. Liu, T. F. Guo, Y. Shi, and Y. Yang, J. Appl. Phys. 89, 3668 (2001).

${ }^{38}$ E. J. W. List, R. Guentner, P. Scanducci de Freitas, and U. Scherf, Adv. Mater. (Weinheim, Ger.) 14, 374 (2002).

${ }^{39}$ During $z-V$ measurements, the actual current is monitored to verify the correct operation of the feedback system. For the measurements in Fig. 7, the injected current was found to exceed the preset value at large negative biases.

${ }^{40}$ R. Rinaldi, R. Cingolani, K. M. Jones, A. A. Baski, H. Morkoc, A. Di Carlo, J. Widany, F. Della Sala, and P. Lugli, Phys. Rev. B 63, 075311 (2001); Appl. Phys. Lett. 82, 70 (2003).

${ }^{41}$ H. Ishii, K. Sugiyama, E. Ito, and K. Seki, Adv. Mater. (Weinheim, Ger.) 11, 605 (1999).

${ }^{42}$ N. Koch, A. Elschner, J. Schwartz, and A. Kahn, Appl. Phys. Lett. 82, 2281 (2003).

${ }^{43}$ N. Koch, A. Kahn, J. Ghijsen, J.-J. Pireaux, J. Schwartz, R. L. Johnson, and A. Elschner, Appl. Phys. Lett. 82, 70 (2003).

${ }^{44}$ W. Brütting, E. Buchwald, G. Egerer, M. Meier, K. Zuleeg, and M. Schwoerer, Synth. Met. 84, 677 (1997).

${ }^{45}$ G. L. J. A. Rikken, Y. A. R. R. Kessener, D. Braun, E. G. J. Staring, and R. Dermandt, Synth. Met. 67, 115 (1994).

${ }^{46}$ L. S. Roman, I. A. Hümmelgen, F. C. Nart, L. O. Péres, and E. L. de Sá, J. Chem. Phys. 105, 10614 (1996).

${ }^{47}$ I. H. Campbell, T. W. Hagler, D. L. Smith, and J. P. Ferraris, Phys. Rev. Lett. 76, 1900 (1996).

${ }^{48}$ I. D. Parker, J. Appl. Phys. 75, 1656 (1994).

${ }^{49}$ E. Ettedgui, H. Razafitrimo, K. T. Park, and Y. Gao, J. Appl. Phys. 75, 7526 (1994).

${ }^{50}$ S. C. Veenstra, Doctoral Dissertation, Univ. of Groningen, The Netherlands, September 2002; www.ub.rug.nl/eldoc/dis/science/ s.c.veenstra/titlecon.pdf

${ }^{51}$ S. M. Tadayyon, K. Griffiths, P. R. Norton, C. Tripp, and Z. Popovic, J. Vac. Sci. Technol. A 17, 1773 (1999).

${ }^{52}$ E. M. Conwell, Synth. Met. 83, 101 (1996).

${ }^{53}$ J. Cornil, D. Beljonne, R. H. Friend, and J. L. Brédas, Chem. Phys. Lett. 223, 82 (1994).

${ }^{54}$ K. Pilcher, D. A. Halliday, D. D. C. Bradley, P. L. Burn, R. H. Friend, and A. B. Holmes, J. Phys.: Condens. Matter 5, 7155 (1993).

${ }^{55}$ U. Rauscher, H. Bässler, D. D. C. Bradley, and M. Hennecke, Phys. Rev. B 42, 9830 (1990).

${ }^{56}$ R. Mahrt, J. Yang, A. Greiner, H. Bäßler, and D. D. C. Bradley, Makromol. Chem., Rapid Commun. 11, 415 (1990).

${ }^{57}$ D. Oelkrug, J. Gierschner, H.-J. Egelhaaf, L. Lüer, A. Tompert, K. Müllen, S. Stalmach, and H. Meier, Synth. Met. 121, 1693 (2001).

${ }^{58}$ D. Beljonne, Z. Shuai, R. H. Friend, and J. L. Brédas, J. Chem. Phys. 102, 2042 (1995).

${ }^{59}$ M. Yu. Lavrentiev, W. Barford, S. J. Martin, H. Daly, and R. Bursill, Phys. Rev. B 59, 9987 (1999).

${ }^{60}$ S. Karabunarliev and R. Bittner, Phys. Rev. Lett. 90, 057402 (2003). 
${ }^{61}$ A. Ruini, M. J. Caldas, G. Bussi, and E. Molinari, Phys. Rev. Lett. 88, 206403 (2002).

${ }^{62}$ S. J. Martin, D. D. C. Bradley, P. A. Lane, H. Mellor, and P. L. Burn, Phys. Rev. B 59, 15133 (1999).

${ }^{63}$ S. J. Martin, H. Mellor, D. D. C. Bradley, and P. L. Burn, Opt. Mater. (Amsterdam, Neth.) 9, 88 (1993).

${ }^{64}$ E. L. Frankevich, A. A. Lymarev, I. Sokolik, F. E. Karasz, S. Blumenstengel, R. H. Baughman, and H. H. Hörhold, Phys. Rev. B 46, 9320 (1992).

${ }^{65}$ M. Scheidler, U. Lemmer, R. Kersting, S. Karg, W. Riess, B. Cleve, R. F. Mahrt, H. Kurz, H. Bässler, E. O. Göbel, and P. Thomas, Phys. Rev. B 54, 5536 (1996).

${ }^{66}$ R. N. Marks, J. J. M. Halls, D. D. C. Bradley, R. H. Friend, and A. B. Holmes, J. Phys.: Condens. Matter 6, 1379 (1994).

${ }^{67}$ V. I. Arkhipov, E. V. Emelianova, S. Barth, and H. Bässler, Phys. Rev. B 61, 8207 (2000).

${ }^{68}$ D. Moses, J. Wang, A. J. Heeger, N. Kirova, and S. Brazovski, Synth. Met. 125, 93 (2002).

${ }^{69}$ A. Mathy, K. Ueberhofen, R. Schenk, H. Gregorius, R. Garay, K. Müllen, and C. Bubeck, Phys. Rev. B 53, 4367 (1996).

${ }^{70}$ R. Östebacka, M. Wohlgennant, M. Shkunov, D. Chinn, and Z. V. Vardeny, J. Chem. Phys. 118, 8905 (2003).
${ }^{71}$ M. Chandros, S. Masumdar, S. Jeglinski, X. Wei, Z. V. Vardeny, E. W. Kwock, and T. M. Miller, Phys. Rev. B 50, 14702 (1994).

${ }^{72}$ J. M. Leng, S. Jeglinski, X. Wei, R. E. Benner, Z. V. Vardeny, F. Guo, and S. Mazumdar, Phys. Rev. Lett. 72, 156 (1996).

${ }^{73}$ J.-W. Van der Horst, P. A. Bobbert, M. A. Michels, and H. Bässler, J. Phys. Chem. 114, 6950 (2001).

${ }^{74}$ P. Gomes da Costa and E. M. Conwell, Phys. Rev. B 48, 1993 (1993).

${ }^{75}$ Z. Shuai, J. L. Brédas, and W. P. Su, Chem. Phys. Lett. 228, 301 (1994).

${ }^{76}$ M. Rolfing and S. G. Louie, Phys. Rev. Lett. 82, 1959 (1999).

${ }^{77}$ M. Knupfer, J. Fink, E. Zojer, G. Leising, U. Scherf, and K. Müllen, Phys. Rev. B 57, R4202 (1998).

${ }^{78}$ M. Wohlgenannt, W. Graupner, G. Leising, and Z. V. Vardeny, Phys. Rev. B 60, 5321 (1999).

${ }^{79}$ Because of the continuity of $\varepsilon_{\mathrm{r}} E$, the field in the vacuum gap is a factor of $\varepsilon_{\mathrm{r}}(=12.9$ for GaAs and 11.7 for $\mathrm{Si})$, larger in the gap than at the semiconductor surface. The dielectric constant of organic semiconductors is around 2-3.

${ }^{80}$ H. Hasegawa, N. Negoro, S. Kasai, Y. Ishikawa, and H. Fulikuwa, J. Vac. Sci. Technol. B 18, 2100 (2000).

${ }^{81}$ L. Libioulle and S. F. Alvarado (unpublished). 